Finn-Rasmus Bull \& Judith Muster

Hierarchie im Spannungsfeld konkurrierender Erwartungen

\section{Journal [ürr Psychologie} 29. Jahrgang - 2021 · Hett 1

Agile Organisationen Versuch einer kritischen Bestandsautrahnme

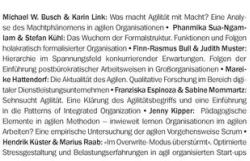

Journal für Psychologie

29. Jahrgang, Nr. 1, 2021, Seite 72-92

Psychosozial-Verlag

DOI: 10.30820/0942-2285-2021-1-72 


\section{Impressum}

Journal für Psychologie

Theorie - Forschung - Praxis

Zeitschrift der Neuen Gesellschaft

für Psychologie (NGfP)

www.journal-fuer-psychologie.de

ISSN (Online-Ausgabe): 2198-6959

ISSN (Print-Ausgabe): 0942-2285

29. Jahrgang, 2021, Heft 1

Herausgegeben von Andrea Birbaumer und Ralph Sichler

https://doi.org/10.30820/0942-2285-2021-1

ISBN der Print-Ausgabe: 978-3-8379-8354-8

ViSdP

Die HerausgeberInnen; bei namentlich

gekennzeichneten Beiträgen die AutorInnen.

Namentlich gekennzeichnete Beiträge stellen nicht in jedem Fall eine Meinungsäußerung der

HerausgeberInnen, der Redaktion oder des Verlages dar.

\section{Herausgebende}

Mag. Andrea Birbaumer, Wien · Dr. Martin Dege, Berlin · Dr. Peter Mattes, Berlin/Wien · Prof. Dr.

Günter Mey, Magdeburg-Stendal/Berlin · Dr. Aglaja

Przyborski, Wien • Paul Sebastian Ruppel, Bochum .

Univ.-Doz. Dr. Ralph Sichler, Wiener Neustadt · Dr.

Anna Sieben, Bochum/Jülich · Prof. Dr. Thomas

Slunecko, Wien

\section{Wissenschaftlicher Beirat}

Prof. Dr. Molly Andrews · Prof. Dr. Thea Bauriedl . Prof. Dr. Jarg Bergold · Prof. Dr. Klaus-Jürgen Bruder • Prof.

Dr. Stefan Busse · Prof. Dr. Tanja Eiselen • Prof. Dr. Jörg Frommer · Prof. Dr. Heiner Keupp · Prof. Dr. Carlos Kölbl · Prof. Dr. Helmut E. Lück · PD Dr. Günter Rexilius · Prof. Dr. Dr. h.c. Wolff-Michael Roth • Prof. Dr. Christina Schachtner - Prof. Dr. Rudolf Schmitt . Prof. Dr. Ernst Schraube • Prof. Dr. Margrit Schreier . Prof. Dr. Hans-Jürgen Seel · Dr. Michael Sonntag . Prof. Dr. Hank Stam · Dr. Irene Strasser, Klagenfurt · Prof. Dr. Dr. Wolfgang Tress • Prof. Dr. Jaan Valsiner - Dr. Barbara Zielke · Prof. Dr. Dr. Günter Zurhorst

\section{Erscheinen}

Halbjährlich als Organ der Neuen Gesellschaft für Psychologie (NGfP) als Open-Access-Publikation und parallel als Print-Ausgabe.

\author{
Verlag \\ Psychosozial-Verlag \\ Walltorstraße 10 \\ D-35390 Gießen \\ info@psychosozial-verlag.de \\ www.psychosozial-verlag.de
}

\section{Abonnentenbetreuung}

aboservice@psychosozial-verlag.de

\section{Bezug}

Jahresabonnement 49,90€ (zzgl. Versand)

Einzelheft 29,90€ (zzgl. Versand)

Studierende erhalten gegen Nachweis 25\% Rabatt, Mitglieder der NGfP erhalten 30\% Rabatt auf den Preis des Jahresabonnements.

Das Abonnement verlängert sich um jeweils ein Jahr, sofern nicht eine Abbestellung bis acht Wochen vor Beendigung des Bezugszeitraums erfolgt.

\section{Anzeigen}

Anfragen richten Sie bitte an den Verlag:

anzeigen@psychosozial-verlag.de

DieZeitschrift Journal für Psychologie wird regelmäßig in der Publikationsdatenbank PSYNDEX des Leibniz-Institut für Psychologie/Leibniz Institute for Psychology (ZPID) erfasst.

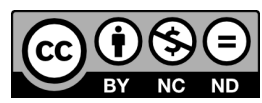

Die Beiträge dieser Zeitschrift sind unter der Attribution-NonCommercial-NoDerivatives 4.0 International Lizenz (CC BY-NC-ND 4.0) lizensiert. Diese Lizenz erlaubt die private Nutzung und unveränderte Weitergabe, verbietet jedoch die Bearbeitung und kommerzielle Nutzung. Weitere Informationen finden Sie unter: creativecommons.org/ licenses/by-nc-nd/4.0/ 


\title{
Hierarchie im Spannungsfeld konkurrierender Erwartungen
}

\section{Folgen der Einführung postbürokratischer Arbeitsweisen in Großorganisationen}

\author{
Finn-Rasmus Bull \& Judith Muster
}

Journal für Psychologie, 29(1), 72-92

https://doi.org/10.30820/0942-2285-2021-1-72

CC BY-NC-ND 4.0

www.journal-fuer-psychologie.de

\section{Zusammenfassung}

Der Beitrag geht der Frage nach, welche Folgen der Einführung postbürokratischer Arbeitsweisen in Großorganisationen sich mit Blick auf den Umgang mit Hierarchie beobachten lassen und wie diese aus organisationssoziologischer Sicht verortet werden können. Ziel ist es, der oft einseitig kritischen Debatte zur Rolle von Hierarchie in Organisationen eine differenzierte Perspektive hinzuzufügen, die die Frage nach Funktionen zum Ausgangspunkt der Analyse macht. Anhand zweier empirischer Fälle wird vor dem Hintergrund äquivalenzfunktionalistischer Annahmen eine solche Analyse durchgeführt. Dabei werden die Bezugsprobleme von Strukturlösungen in Form formaler wie auch informaler Hierarchie identifiziert sowie ihre jeweiligen Folgeprobleme nachgezeichnet. Deutlich wird, dass die Realisierung postbürokratischer Arbeitsweisen in den untersuchten Fällen durch die Entstehung von Spannungsfeldern zwischen konkurrierenden Erwartungsstrukturen sowohl an organisationalen Schnittstellen als auch innerhalb der postbürokratischen Einheiten geprägt ist. Der Beitrag schließt mit einem kurzen Resümee und Ausblick zu möglichen Anschlüssen an die hier vorgeschlagene Perspektive.

Schlüsselwörter: Postbürokratie, Hierarchie, Organisationssoziologie, Äquivalenzfunktionalismus, funktionale Analyse

\section{Summary}

Hierarchy in the field of tension between competing expectations

Consequences of the introduction of post-bureaucratic organizing in large-scale organizations

This article explores the question of what consequences of the introduction of post-bureaucratic organizing in large-scale organizations can be observed regarding the handling of 
hierarchy and how these can be located from an organizational sociological point of view. Our goal is to add a differentiated perspective to the often one-sidedly critical debate on the role of hierarchy in organizations, making the question of functionality the starting point of the analysis. Based on two empirical cases, such an analysis is carried out against the background of equivalence-functionalist assumptions. In doing so, we point out the problems for which formal and informal hierarchy are identified as structural solutions. Furthermore, we show which consequences result from these problem/solution constellations. It becomes clear that the realization of post-bureaucratic working methods in the cases studied is characterized by the emergence of fields of tension between competing expectation structures both at organizational interfaces and within the post-bureaucratic units. The article concludes with a short summary and outlook on possibilities for further research building on the perspective proposed here.

Keywords: Post-bureaucracy, hierarchy, organizational sociology, equivalence-functionalism, functional analysis

\section{$1 \quad$ Einleitung}

Das Label Agilität prägt seit einiger Zeit die Managementdiskurse rund um modernes Organisieren. Mit den entsprechenden Konzepten werden eine ganze Reihe von Hoffnungen verbunden, die je nach Situation, Lesart und Interessenlage von gesteigerter Innovationsfähigkeit über Effizienzgewinne bis hin zur Demokratisierung von Unternehmen reichen. Der Begriff der Agilität bleibt dementsprechend häufig inhaltlich unterbestimmt und gewinnt nicht zuletzt genau daraus als »flottierender Signifikant « (Ortmann 2017), also durch seine Mehrdeutigkeit und inhaltliche Varianz, in der Praxis an Anschlussfähigkeit (siehe auch Kieser 1996).

Eine empirische Annäherung an Praktiken agilen Organisierens ist dementsprechend damit konfrontiert, eine diffuse Gemengelage vorzufinden. Von vorranging legitimitätsstiftenden Bekundungen in Richtung relevanter Umwelten bis hin zu weitreichenden Umgestaltungen innerorganisationaler Prozesse ist das empirische Untersuchungsfeld ebenso weitläufig wie divers. Aller Heterogenität zum Trotz lassen sich in den korrespondierenden Diskursen um Agilität aber typische Annahmen und Stoßrichtungen identifizieren, die Organisationspraktiker*innen ebenso wie Organisationsforscher*innen in wiederkehrenden Zyklen verstärkt beschäftigen. Ein gemeinsamer Nenner ist dabei der Abbau formaler Hierarchie (Kühl 2015, 62).

Losgelöst von der Frage, ob es sich bei Agilität nur um einen weiteren Fall von »Moden \& Mythen des Organisierens « (Kieser 1996) handelt, ist die Reduktion von formaler Hierarchie ein potenziell markanter Eingriff in bestehende Organisations- 
gefüge. Denn aller Rhetorik insbesondere der Managementliteratur zum Trotz stellt Hierarchie weiterhin ein zentrales Strukturmerkmal von Organisationen dar (Kühl 2011, 69-88). Welche Effekte stellen sich empirisch also ein, wenn der Verzicht auf bzw. die Reduktion von formaler Hierarchie als Strukturmerkmal realisiert wird? Und wie stellt sich der faktische Umgang mit neuen Kooperationsmodellen in der Organisationspraxis dar?

Diesen Leitfragen widmet sich der folgende Beitrag aus einer dezidiert organisationssoziologischen Perspektive, die Organisationen als Systeme mit einer besonderen Eigenlogik versteht (Luhmann 1995). Dazu gehört auch, dass wir uns im Rahmen unserer Beschreibung einer deskriptiven Darstellung verpflichtet sehen, die nicht auf zu erreichende Idealzustände des Organisierens abzielt. Zudem gilt unser Blick vorrangig organisationalen Strukturen und weniger den handelnden Personen selbst. Das schließt natürlich nicht aus, die aus der Struktur resultierenden Handlungen sowie Effekte auf Personengruppen zum Thema zu machen.

Die Argumentation erfolgt dabei entlang von zwei Stützpfeilern, die unsere Überlegungen leiten und prägen. So werden wir erstens empirische Einsichten aus einem Forschungsprojekt zu »Postbürokratischem Organisieren in Großorganisationen « vorstellen, das sich mit der Einführung postbürokratischer Arbeitsweisen ${ }^{1}$, unter anderem in Form postbürokratischer Inseln in Unternehmen, beschäftigt hat. Wir nutzen zudem zweitens die funktionale Analyse als eine Heuristik, die Organisationsstrukturen als Ausprägung spezifischer Problem/Lösungs-Schemata in den Mittelpunkt rückt und diese so auch mit Blick auf Alternativen kritisch würdigen kann. Bevor wir in die Analyse gehen, werfen wir aber zunächst einen Blick auf die etablierte Hierarchiekritik, die den Diskurs rund um postbürokratische Arbeitsweisen prägt und der wir eine neue Perspektive hinzufügen wollen.

\section{Hierarchiekritik und Postbürokratie}

Diskurse rund um postbürokratische Arbeitsweisen fußen auf einer langen Tradition der Bürokratiekritik. ${ }^{2}$ Während die Label der korrespondierenden Modelle und Konzepte dabei in Wissenschaft und Praxis über die Zeit wechseln (Kühl 2015, 10), lassen sich übergreifend drei Merkmale identifizieren, die wiederkehrend eine prägende Rolle bei der Konzeption postbürokratischer Modelle spielen: Entdifferenzierung als Auflösung etablierter Arbeitsteilung, Enthierarchisierung und Dezentralisierung in Form von gesteigerter Autonomie einzelner Organisationseinheiten (ebd., 60ff.). Dabei ist insbesondere der Verzicht auf bzw. die Ablehnung von formaler Hierarchie ein zentraler Baustein postbürokratischer Modelle. Die entsprechenden Diskurse gehen daher mit einer Hierarchiekritik einher, die sich in aller Regel entlang dreier Argumentationsachsen 
entfaltet. So wird Hierarchie (1) in sachlicher Hinsicht als ineffizient, (2) in normativer Hinsicht als kritikwürdig und (3) mit Blick auf Zeitdiagnosen, die vor allem auf veränderte Umweltbedingungen abzielen, als inadäquat beschrieben. Diese gängigen Einflugschneisen der Kritik werden im Folgenden kurz skizziert. Daran anschließend formulieren wir ein Plädoyer für eine äquivalenzfunktionalistische Perspektive in der Debatte.

\subsection{Einflugschneisen der Hierarchiekritik}

Ein dominanter Strang gängiger Bürokratie- und Hierarchiekritik orientiert sich daran, hierarchische Organisationsstrukturen entlang von Ineffizienzen zu beschreiben. ${ }^{3} \mathrm{Im}$ Zentrum stehen Sachdiagnosen zu Defiziten rationalen oder effizienten Handelns in bürokratischen Organisationen. Die Kritik geht davon aus, dass hierarchische Kontrolle und Steuerung hohe Transaktionskosten auslösen. So entstünden durch Überwachungsund Kontrollaufgaben Ineffizienzen, die zusätzliche Kosten generieren und Ressourcen binden würden (Seabright und Delacroix 1996, 141f.). Auch lange Kommunikationsund Entscheidungswege, die sich aus dem hierarchischen Aufbau von Organisationen ergeben würden, werden als Ineffizienzen identifiziert, da diese, so die Diagnose, Entscheidungen verlangsamen und Informationen nur unzureichend durch die Organisation leiten (Moser 2017, 29ff.; siehe auch die Darstellung bei Oberg und Walgenbach 2007, 171ff.) sowie Veränderungen hemmen würden (Altherr 2019, 417f.). Letzteres wird dabei insbesondere auch mit Blick auf die Innovations- und Adaptionsfähigkeit von Organisationen thematisiert. Hierarchische Strukturen seien vor allem für die effiziente Abarbeitung von Routineaufgaben geeignet, während sie mit Blick auf die Hervorbringung von Innovationen limitiert blieben, da die dafür notwendige Kollaboration nur unzureichend abgebildet werden könne (Adler 2001, 216). Sie würden sich daher vor allem für Organisationen eignen, die in stabilen Umwelten operierten (Burns und Stalker 1971, 119ff.).

Heckscher (1994) zufolge griffen nicht wenige dieser Befunde allerdings zu kurz. Vor dem Hintergrund der Entwicklung eines Idealtyps postbürokratischen Organisierens gelte es $\mathrm{zu}$ unterscheiden, »which aspects of these problems are results of badly managed bureaucracy, and which are inherent in the model « (ebd., 18; Herv. im Orig.). So seien Entbürokratisierungsbemühungen nicht selten Versuche, bürokratische Strukturen besser zu nutzen als sie zu überwinden (ebd., 19f.). Auch Hales (2002, 52f.) weist darauf hin, dass sich die Kritik an Dysfunktionalitäten bürokratischer Prinzipien wie unter anderem Hierarchie häufig auf das Ausmaß ihrer Nutzung und nicht auf die Prinzipien selbst beziehe. Darüber hinaus, so Heckscher, gäbe es aber drei grundlegende Probleme hierarchisch-bürokratischer Strukturen, denen postbüro- 
kratisches Organisieren begegnen soll. Erstens sei infolge festgelegter Arbeitsteilung die Möglichkeit, das Wissen von Organisationsmitgliedern nutzbar zu machen, zu stark eingeschränkt. Stattdessen würde dieses lediglich nach Zuständigkeit einbezogen, wodurch die Organisation in dieser Hinsicht limitiert bliebe. Zweitens sei in bürokratischen Organisationen die Möglichkeit der Einflussnahme auf und Strukturierung von informalen Prozessen und Aushandlungen durch die etablierten Kontrollmechanismen zu gering, um diese für die Organisation sinnvoll auszurichten. Drittens wird diagnostiziert, dass durch die hierarchische Ordnung und die Zuständigkeit für Strategiefragen auf den oberen Ebenen sowie durch Abteilungsgrenzen Wandel zu punktuell und intensiv erfolge, wodurch hohe Reibungsverluste entstünden (Heckscher 1994, 20ff.).

Schon diese kurze Übersicht macht deutlich: Hierarchisch-bürokratischen Organisationen wird hinsichtlich effizienter und flexibler Steuerung oft ein negatives Zeugnis ausgestellt. Darüber hinaus prägt die gängige Hierarchiekritik ein zweiter, stärker normativ ausgerichteter Strang, der sich an Wertvorstellungen guten Organisierens misst. Formale Hierarchie ist diesen Annahmen zufolge nicht nur eine wenig erfolgversprechende Gestaltungsformel von Organisationen, sie wird darüber hinaus vor dem Hintergrund normativer Annahmen kritisiert. Mitarbeitende seien in hierarchischen Unternehmen qua System ohnmächtig und fremdbestimmt, wodurch zudem demokratische Prinzipien eingeschränkt werden würden. Die daraus resultierenden Konflikte würden sich, so zuweilen die Diagnose, aufgrund veränderter Wertvorstellungen zunehmend verschärfen (Moser 2017, 32ff.). ${ }^{4}$ Hierarchie als Form » einer direkten Steuerung des Gegenübers $[\ldots]$ « könne gar »einer Entwürdigung der Person « gleichkommen (Altherr 2019, 423).5 Ansätze und Ideen reduzierter formaler Hierarchien werden daher nicht zuletzt auch unabhängig von rationalen Effizienzerwägungen unter Aspekten der Steigerung von Partizipation und Demokratisierung von Organisationen diskutiert (Rothschild-Whitt 1979; vgl. auch Ames 1995). In der diskursprägenden Managementliteratur werden Formen des postbürokratischen Organisierens gar als Ausdruck fortschreitender Evolution des menschlichen Bewusstseins postuliert (Laloux 2014).

Begleitet werden beide Stränge der Hierarchiekritik üblicherweise von Zeitdiagnosen, die die Abnahme oder zumindest den Wandel von Hierarchie in Organisationen als unvermeidlich erscheinen lassen. Die Zeitdimension des Diskurses wird insbesondere dann deutlich, wenn auf veränderte oder sich verändernde Umweltbedingungen rekurriert wird. Organisationen, so die Annahme, stünden aus verschiedenen Richtungen unter Veränderungsdruck. Als Auslöser werden dabei unterschiedliche Entwicklungen in den relevanten Organisationsumwelten ausgemacht. Die Diagnosen reichen dabei von gesteigerter Unsicherheit infolge von Digitalisierung (Altherr 2019) oder neuer Wettbewerbs- und Marksituationen (Reihlen 1998, 3ff.; Heydebrand 1989) bis hin zur grundlegenden Transformation des Wirtschaftssystems von physischen Produkten 
hin zu einer »information-intensive economy« (Child und McGrath 2001, 1144). Auch werden gewandelte Erwartungen und Bedarfe neuer Generationen von Mitarbeitenden (Clegg 2012, 77ff.; Lee und Edmondson 2017, 37) sowie unterschiedliche Megatrends wie Globalisierung, eine erhöhte Zugänglichkeit von Informationen oder Individualisierung (Moser 2017, 27-39) als Auslöser für die Notwendigkeit von Veränderungsprozessen in Organisationen identifiziert. ${ }^{6}$ Der Reduktion formaler Hierarchie wird für diese Anpassungsprozessen eine zentrale Rolle zugeschrieben. Hierarchie als Koordinationsmechanismus, so die Annahme, könne sowohl nach außen nicht schnell genug auf sich verändernde Umweltbedingungen wie beispielsweise Anforderungen von Kund*innen reagieren, als auch nach innen eine verstärkte Abhängigkeit vom Wissen der Mitarbeitenden auf allen organisationalen Ebenen nicht ausreichend bearbeiten (Lee und Edmondson 2017, 37).

Diese drei Stränge der Hierarchiekritik sind nicht selten miteinander verzahnt. Ihre Trennung soll an dieser Stelle vor allem deutlich machen, dass ihren Einflugschneisen unterschiedliche Perspektiven zugrunde liegen: die Orientierung an Effizienzkriterien, die normative Setzung von Annahmen guten Organisierens sowie die Unterstellung der Notwendigkeit der Anpassung an bestimmte gesellschaftliche Entwicklungen. Alle drei Einflugschneisen der Kritik weisen dabei auf wichtige Rahmenbedingungen der Ausgestaltung formaler Hierarchie in Organisationen hin. Gerade deshalb lohnt es, ihnen eine kritische Würdigung hierarchischer Mechanismen gegenüberzustellen, die vor dem Hintergrund einer systemtheoretisch-organisationssoziologischen Perspektive auch ins Kalkül zieht, für welche Probleme Hierarchie als Lösung auftritt, anstatt Hierarchie vorrangig als Problemauslöser zu begreifen.

\subsection{Plädoyer für eine äquivalenzfunktionalistische Perspektive}

Dass Hierarchie Kritik auf sich zieht, ist aus einer gesellschaftlichen Perspektive zunächst wenig verwunderlich. Schließlich zeichnet sich die moderne, funktional differenzierte Gesellschaft gerade dadurch aus, dass Hierarchie in allen gesellschaftlichen Bereichen außer in Organisationen - als primäres Strukturationsprinzip ersetzt wurde (Kühl 1999, 3; Fuchs 2009, 60ff.). Und auch die Kritik selbst ist sicher nicht an jeder Stelle von der Hand zu weisen. Insbesondere normative wie auch zeitdiagnostische Einflugschneisen der Hierarchiekritik laufen allerdings Gefahr, einen blinden Fleck zu erzeugen, den wir im vorliegenden Beitrag ins Zentrum rücken wollen: die Funktionalität formaler Hierarchie.

Denn die empirische Realität in Organisationen ist deutlich komplexer als solche Perspektive es nahelegen. Organisationen sind immer auch geprägt von Zielkonflikten und Zweckwidersprüchen, die eine eindeutig richtige oder adäquate Konfiguration 
der eigenen Verfasstheit de facto verunmöglichen (March und Simon 1958; Luhmann 1973, 227-236). Die Frage, ob und in welcher Form formale Hierarchie in Organisationen genutzt werden kann und sollte, ist daher vor allem eine Frage ihrer Funktionalität. Die faktische Realisierung postbürokratischer Arbeitsweisen in Organisationen entscheidet sich dementsprechend immer auch daran, ob die Funktionen bisheriger oder bisher gängiger Strukturen, also auch formaler Hierarchie, in der neuen organisationalen Konfiguration weiterhin sichergestellt oder substituiert werden können (vgl. Ingvaldsen und Benders 2020; Oedzes et al. 2019). Erst wenn Strukturen als funktionale Äquivalente in den Blick genommen werden, werden somit tatsächliche Potenziale alternativer Organisations- und Arbeitsweisen sichtbar. Ebendiese, stärker auf binnenorganisationale Problemstellungen als auf organisationsexterne Faktoren ausgerichtete Perspektive scheint in der gängigen Hierarchiekritik zu häufig unterrepräsentiert (siehe aber Heckscher 1994). Entsprechend gilt es, den Abbau formaler Hierarchie daraufhin zu prüfen, inwieweit tatsächlichen innerorganisationalen Bezugsproblemen weiterhin Rechnung getragen wird:

»Vielleicht lässt sich in der Tat eine gewisse Tendenz zur Abschwächung der hierarchischen Orientierung durch Sachzwänge und horizontale Interdependenzen beobachten. Diese Erweiterung des Blicks ist von Wert, aber sie wird auf die Dauer nicht als Kritik der Hierarchie, sondern nur durch ein genaueres Verständnis ihrer Funktion fruchtbar sein « (Luhmann 2018a, 237; Herv. im Orig.).

Daher wollen wir, anstatt wie die gängige Hierarchiekritik bereits an Diagnosen zu Folgeproblemen formaler Hierarchie anzusetzen, zunächst die Funktionalität hierarchischer Organisationsstrukturen in den Blick nehmen. Das schließt die Beschreibung von Problemen hierarchischer Steuerung nicht aus. Zunächst gilt es aus unserer Sicht aber, ihre übergeordneten Bezugsprobleme zu identifizieren. So erscheinen beispielsweise kritische Diagnosen zur Filterung von Informationsflüssen durch Hierarchieebenen in einem neuen Licht, wenn ebendiese Filterfunktion als Lösung für das Bezugsproblem überbordender Komplexität und daraus folgender Handlungsunfähigkeit von Entscheidungsträger*innen identifiziert werden kann (Kühl 2001a, 483).

Aus einer äquivalenzfunktionalistischen Perspektive sind unterschiedliche Herangehensweisen und Strukturformen, sofern sie jeweils in der Lage sind, ein bestimmtes Bezugsproblem zu lösen, mit Blick auf dieses Bezugsproblem zunächst gleichrangig. In der organisationalen Praxis zeigt sich dabei schnell, dass mit Kombinationen unterschiedlicher Problemlösungsstrukturen zu rechnen ist - nicht zuletzt deshalb, weil jede Organisation neben der entschiedenen Formalstruktur auch auf die Herausbildung informaler Strukturen angewiesen ist (Luhmann 1995; für einen Überblick siehe auch von Groddeck und Wilz 2015). Ebendiese, häufig gar funktionale, Gleichzeitigkeit un- 
terschiedlicher organisationaler Erwartungsstrukturen droht durch eine Verengung des Diskurses auf Ineffizienzen, Wertvorstellungen und Zeitdiagnosen aus dem Blick zu geraten. Das Potenzial einer solchen Betrachtung wollen wir im Folgenden anhand empirischer Beobachtungen aufzeigen.

\section{Folgen der Einführung postbürokratischer Arbeitsweisen in Großorganisationen}

Um einen äquivalenzfunktionalistischen Blick einnehmen zu können, dient uns die funktionale Analyse als Heuristik. Sie basiert auf der Idee, dass sich Strukturen als Lösungen eines jeweils spezifischen Bezugsproblems ausbilden. Da die empirisch vorfindbaren Lösungen kontingent sind (auch dann, wenn sie in der konkreten Organisation nicht als kontingent wahrgenommen werden), ist die funktionale Analyse in erster Linie eine Methode, funktional äquivalente Lösungsmöglichkeiten für bestimmte Bezugsprobleme aufzuzeigen. Bezugsprobleme können dabei sowohl theoretisch abgeleitet als auch empirisch generiert werden (Luhmann 2005). Die funktionale Analyse zielt darauf $\mathrm{ab}$ aufzuschlüsseln, welche Strukturen sich empirisch als Lösungen realisieren, welche Effekte und Folgeprobleme sie zeitigen und inwiefern sie andere Strukturlösungen beschränken, ermöglichen oder stabilisieren. Damit würdigt die funktionale Analyse die jeweils aktuelle Normsetzung kritisch, nämlich hinsichtlich anderer Problem-/Lösungskonstellationen (Luhmann 1995, 19). Kette und Tacke $(2015,244)$ bezeichnen sie daher auch als »Entdeckungsverfahren für Strukturalternativen «.

Bei der Untersuchung typischer Problem-/Lösungskonstellationen postbürokratischen Organisierens steht man dabei vor einer besonderen Herausforderung. In der Praxis lassen sich postbürokratische Organisationen in Reinform nämlich kaum beobachten (Alvesson und Thompson 2006; vgl. auch Hales 2002), insbesondere dann, wenn von Kleinstorganisationen wie Start-ups abgesehen wird, die durch ihre geringe Größe vielfach noch verstärkt durch Gruppenmechanismen als durch die üblichen Logiken organisierter Sozialsysteme geprägt sind (vgl. Kühl 2002). Abgesehen von Idealtypen postbürokratischen Organisierens finden sich aber vielerorts Bestrebungen, postbürokratische Modelle und Mechanismen parallel oder ergänzend zur klassischen Organisationsstruktur zu integrieren (Foss 2003, 346; Alvesson und Thompson 2006, 500f.). Die praktische Realisierung findet also nicht selten im Umfeld weiterhin klassisch aufgestellter Organisationen statt (siehe auch Heydebrand 1989, der in seiner Konzeption neuer, postbürokratischer Organisationsformen diese vorrangig als kleine Organisationen oder als kleinere Einheiten größerer Organisationen beschreibt). Gerade in solchen Fällen ist dabei zu erwarten, dass der Umgang mit formaler Hierarchie ein kontestiertes Feld der organisationalen Praxis darstellt. 
Um uns diesem Feld analytisch zu nähern, stützen wir uns im Folgenden auf zwei empirische Fälle, die von den Autor*innen im Rahmen eines Forschungsprojekts erhoben wurden und die uns im Folgenden als Ausgangspunkt und Illustration der Beschreibung von Folgen der Einführung postbürokratischer Arbeitsweisen dienen sollen. ${ }^{7}$ Bei den beforschten Einheiten handelt es sich um postbürokratische Inseln in Großorganisationen, die in der Folge neben bereichsinternen Themen zusätzlich damit konfrontiert sind, die Anschlussfähigkeit an die sie umgebene Gesamtorganisation aufrechterhalten zu müssen. Dadurch geraten über bereichsinterne Effekte hinaus insbesondere auch Herausforderungen des Schnittstellenmanagements zur organisationsinternen Umwelt in den Blick.

Zur Einordung der beiden Fälle stellen wir beide in aller gebotenen Kürze dar. Bei den beforschten Unternehmen handelt es sich um einen französischen Werkzeughersteller, AGILETools SE, und ein britisches Softwareunternehmen, FlatSoft Ltd., in deren weitgehend klassisch organisierten Gesamtorganisationen einzelne Bereiche auf Basis postbürokratischer Arbeitsweisen reorganisiert oder integriert wurden. ${ }^{8}$

In einem Werkzeugmaschinenwerk der AGILETools SE sollte die bisher extern eingekaufte Spanntechnik fortan intern gefertigt werden, um Fertigungsstunden und Arbeitsplätze zu sichern und die Produktivität zu erhöhen. Weil ein klassisches Projektmanagement für das Insourcing-Projekt schon in einer frühen Phase zu scheitern drohte, wurde stattdessen verstärkt auf eine Einbeziehung der Mitarbeitenden gesetzt. Nachdem das selbstorganisierte Insourcing-Projekt erfolgreich verlief, beschloss die Projektleitung, auch die solchermaßen konzipierte Fertigung nach Prinzipien der Selbstorganisation arbeiten zu lassen. Das Ergebnis war die sogenannte selbstorganisierte Halle, in der Einzelteile der Spanntechnik in sogenannten Assemblages gebaut wurden: selbstorganisierte Gruppen mit unterschiedlichen Gewerken, die gemeinsam bestimmte Teile der Spanntechnik fertigten. Innerhalb der Assemblages sollten die Mitarbeitenden mit Blick auf ihr spezifisches Bauteil breit qualifiziert werden, um eine höhere, produktorientierte Flexibilität zu erreichen. ${ }^{9}$ Zudem sah das Hallenkonzept vor, eine, für die AGILETools SE untypische, cross-funktionale Hallenleitung sowie anstelle von Vorarbeitern sogenannte Rep Links für die Assemblages zu etablieren, die den Informationsfluss zwischen Assemblage und Hallenleitung sicherstellen sollten, aber ohne formale Weisungsbefugnis agierten. Die Erhebung fand vier Jahre nach Einführung der selbstorganisierten Halle statt und umfasste 21 Interviews mit Mitarbeitenden und Führungskräften, die entweder am Einführungsprozess beteiligt oder zum Zeitpunkt der Gespräche innerhalb der Halle beschäftigt waren.

Bei der FlatSoft Ltd. wurde die interne Organisationsentwicklung im Zuge einer umfassenden Restrukturierung auf das Organisationsmodell Holacracy umgestellt, das sich von althergebrachten Abteilungsstrukturen löst und stattdessen auf sogenannte Kreise entlang von Arbeitszusammenhängen setzt. Auslöser für die Organisationsveränderung 
war unter anderem die Diagnose des neuen Abteilungsleiters, dass sich die einzelnen Bereiche zunehmend zu abgeschotteten Silos entwickelt hätten. Durch die Einführung von Holacracy sollte cross-funktionale Kooperation sichergestellt und die bestehende Silostruktur aufgelöst werden. Dazu wurde die Abteilung in neu formierten Kreisen entlang zentraler Aufgaben und Arbeitszusammenhänge organisiert. In diesen Kreisen waren Mitarbeitende mit unterschiedlichen Rollen, zum Teil in mehreren Kreisen, vertreten. Innerhalb der Teams nahmen, dem Modell folgend, sogenannte Lead Links eine zentrale Position ein. Sie sollten für das jeweilige Team vor allem Koordinationsfunktionen, unter anderem die Besetzung offener Rollen, übernehmen. Im Gegensatz zu klassischen Führungskräften war die Weisungsbefugnis der Lead Links allerdings stark begrenzt. Stattdessen wurden Entscheidungen entweder im Rahmen einer erweiterten Autonomie einzelner Rollen oder über unterschiedliche Meeting-Formate getroffen. In diesen Tactical und Governance Meetings wurden sowohl operative Entscheidungen zwischen Rolleninhaber*innen als auch grundlegendere, die Organisationsstrukturen betreffenden, Entscheidungen getroffen. Als Ersatz für formale Hierarchie traten in dieser Hinsicht demnach eine stärker dezentralisierte Entscheidungsautonomie auf Ebene der einzelnen Rollen sowie die rollenübergreifende Abstimmung in stark vorstrukturierten und moderierten Interaktionsformaten auf (für eine detaillierte Darstellung des Modells siehe Robertson 2015; für eine Diskussion des Modells am Beispiel des Vorreiterunternehmens Zappos siehe Bernstein et al. 2016). Insgesamt wurden bei der FlatSoft Ltd. zwölf Interviews mit Mitarbeitenden, Lead Links und dem Abteilungsleiter der Organisationsentwicklung geführt. Zum Zeitpunkt der Erhebung arbeitete die Abteilung seit anderthalb Jahren im holakratischen Modell, wobei dessen zeitnahe Abschaffung zugunsten einer übergeordneten Reorganisation bereits beschlossen war.

Beide Fälle eint, dass den agierenden Teams bzw. Assemblages durch die Reduktion formaler Hierarchie und neue Formen der Kooperation weitergehende Autonomie eingeräumt werden sollte. Koordination und Steuerung sollten verstärkt durch Mitarbeitende selbst erfolgen. Im Folgenden widmen wir uns der Frage, welche Effekte die Einführung der jeweiligen Modelle mit Blick auf den Umgang mit Hierarchie zeitigte. In der kontrastierenden Betrachtung der Fälle geraten dabei zwei Phänomene besonders in den Blick: die Entstehung konkurrierender formaler Erwartungen an Schnittstellen zwischen Bereich und innerorganisationaler Umwelt (3.1) sowie die informale Reetablierung von Hierarchie innerhalb der postbürokratisch organisierten Bereiche (3.2).

\subsection{Konkurrierende formale Erwartungen an organisationalen Schnittstellen}

Eine erste Folge der Einführung postbürokratischer Arbeitsweisen in Großorganisationen mit Blick auf den Umgang mit formaler Hierarchie zeigt sich im Aufkommen von 
Konkurrenzverhältnissen unterschiedlicher Erwartungsstrukturen. Eine solche Konkurrenz kann sich in zweierlei Hinsicht etablieren. Erstens sind Organisationen bekanntlich immer durch eine doppelte Struktur geprägt, die ihre Mitglieder gleichermaßen mit Verhaltenserwartungen konfrontiert. Zum einen die Formalstruktur der Organisation, die sich aus den offiziellen Organigrammen, Regeln und Vorgaben speist, zum anderen die Informalität, die sich - stets in Bezug auf die formalen Strukturen - in einer Organisation mit der Zeit herausbildet (Kühl 2011, 113-136). Zweitens aber steht die Formalstruktur selbst unter einem gewissen Konsistenzzwang. Formale Erwartungen dürfen sich nicht oder zumindest nicht allzu offensichtlich widersprechen, ansonsten ginge ihr Potenzial zur Orientierung von Handlungen verloren: »In ein formales System können [...] nur Erwartungen aufgenommen werden, die zumindest miteinander verträglich sind, d. h. erfüllt werden können, ohne daß dabei offen gegen andere formale Erwartungen verstoßen werden muß« (Luhmann 1995, 63). Auch hier kann es, treten Widersprüche zutage, also zu Konkurrenzsituationen zwischen formalen Erwartungen kommen. Für die Einführung von Arbeitsweisen in einzelnen Bereichen von Großorganisationen, die in ihrer internen Logik stark von der Restorganisation oder zumindest der für sie relevanten Außenkontakte abweichen, bedeutet dies, dass die Aufrechterhaltung von Anschlussfähigkeit an ihren Schnittstellen ein besonderes Bezugsproblem darstellt. Unter Bedingungen formalisierter Mitgliedschaft kann Kooperation legitimerweise erwartet werden, auch dann, wenn die kooperierenden Bereiche stark divergierende interne Strukturierungen aufweisen. An den übergaberelevanten Punkten muss die Formalstruktur daher Möglichkeiten abbilden, sich sowohl nach außen, in die Organisation hinein, als auch nach innen in Richtung des eigenen Bereichs richtig zu verhalten. Klassischerweise wird die Übergabe an den Schnittstellen in Organisationen qua Hierarchie und funktionaler Arbeitsteilung geregelt: Ansprechpartner*innen ergeben sich aus der Aufbauorganisation, nächste gemeinsame Vorgesetzte sind für den Konfliktfall bekannt. Wird in einzelnen Bereichen aber ganz oder weitestgehend auf die Festlegung formaler Hierarchien oder auf eine zueinander passende Aufteilung von Aufgaben und Verantwortlichkeiten verzichtet, stellt sich die Frage des Schnittstellenmanagements neu.

In den von uns untersuchten Fällen zeigt sich dabei, dass an den Schnittstellen die Passung zwischen postbürokratischen und eher klassisch aufgestellten Bereichen nicht immer gewährleistet werden konnte. Stattdessen etablierten sich konkurrierende formale Erwartungen insbesondere hinsichtlich der Rolle von Hierarchie, die die jeweiligen Logiken repräsentierten.

Im Falle der AGILETools SE zeigte sich diese Dualität in Gestalt der cross-funktionalen Hallenleitung. Diese sollte produktionsnahes Entscheiden und damit kürzere Abstimmungswege gewährleisten. Die unterschiedlichen Funktionen wie Sales, Disposition, Fertigungsleitung und Qualität wurden zu diesem Zweck zwar innerhalb eines Büros in der Halle zusammengeführt, die formalen Berichtslinien in ihre Funk- 
tionsbereiche blieben aber bestehen. Im gemeinsamen Büro entstand eine kooperative Zusammenarbeit, Entscheidungen zu den Produktionsprozessen in der Halle wurden vermehrt in enger Abstimmung zwischen den Funktionen getroffen. Hinzu kam, dass auch Funktionsvertreter*innen, die formal den Mitarbeitenden der Assemblages nicht vorgesetzt waren, verstärkt direkt auf diese zugingen und steuernd in die Arbeitsabläufe eingriffen: »Eigentlich sollen nur die Teamleiter Anweisungen an die Fertigung geben, wir haben das bei uns aber aufgeweicht « (Mitglied der Hallenleitung). Außerhalb der Halle war diese Art der Kooperation zwischen den Abteilungen allerdings sonst eher unüblich. Dadurch gerieten die Mitglieder der Hallenleitung zunehmend in Loyalitätskonflikte mit ihren Vorgesetzten, da die getroffenen Entscheidungen nicht nur gelegentlich die formalen Befugnisse überschritten, sondern zudem von einzelnen Mitgliedern der Hallenleitung gegen die Interessen der eigenen Abteilung getroffen bzw. mitgetragen wurden: »Außerhalb [der Hallenleitung] haben wir unsere Chefs. Wir treffen viele Entscheidungen selbst. Dafür bekommen wir auch mal Haue, aber wir sind als Team stark genug. Wir müssen immer überlegen, wessen Interessen wir vertreten. Auch mal Technischer Service, obwohl wir eigentlich Fertigung sind « (Mitglied der Hallenleitung). Die Konkurrenz der Erwartungsstrukturen zeigt sich hier darin, dass seitens der Hallenleitung, als vermittelnder Instanz zwischen Fertigungsbereich und Linienorganisation, unterschiedliche Ordnungen nach innen und nach außen repräsentiert werden mussten. Zum einen galt es das Bezugsproblem zu lösen, in Richtung der Halle gemeinsame Entscheidungsfähigkeit trotz unterschiedlicher lokaler Rationalitäten (Cyert und March 1963), die sich aus der Funktionszugehörigkeit ergeben, herzustellen. Dies gelang der Hallenleitung durch die sich über die Zeit etablierte Praktik, vorrangig konsensual zu entscheiden und in Richtung der Mitarbeitenden der Halle verstärkt als funktionenübergreifende hierarchische Einheit zu agieren. Als Folgeproblem entstand für die Vorgesetzten der Linienorganisation der Bedarf, die darüberliegende Formalordnung von Zeit zu Zeit zu aktualisieren, damit diese nicht an Gültigkeit einbüßt (vgl. analog am Beispiel formaler Regeln Luhmann 1995, 308). Während berichtet wurde, dass der Hallenleitung seitens der Linienorganisation durchaus Freiräume gewährt wurden, intervenierte diese von Zeit zu Zeit, beispielsweise bei der Überschreitung formaler Kompetenzen hinsichtlich Entscheidungen zur Vergabe von Fertigungsstunden an Zulieferer. Die Entscheidungsfindung zwischen den Funktionen innerhalb der Hallenleitung sowie insbesondere zwischen der Hallenleitung und der Linienorganisation musste daher kontinuierlich verhandelt werden. Daraus ergibt sich ein weiteres, nicht-intendiertes Folgeproblem der Einführung der cross-funktionalen Hallenleitung. Dadurch, dass die formale Zuordnung zur Linienorganisation die Orientierung an der eigenen Funktion nahelegt und diese aus Sicht der Gesamtorganisation vorrangig vor anderen Erwartungen legitimiert ist, liegt das Risiko dieser Aushandlungen in der Konsequenz aufseiten der Hallenleitung (vgl. zur persönlichen 
Zurechnung von Führung jenseits von formaler Hierarchie Muster et al. 2020). Die Durchsetzung der formalen Erwartung der postbürokratischen Einheit, dass die Hallenleitung cross-funktional und autonom agieren sollte, blieb auf diese Weise abhängig von der Bereitschaft der Hallenleitung, entgegen konkurrierender Erwartungen der Linienorganisation ins persönliche Risiko zu gehen, und damit fragil.

Auch bei der FlatSoft Ltd. spielte die Anschlussfähigkeit an die über- und nebengeordnete Struktur der formalen Hierarchie und die damit einhergehende Konkurrenz von Erwartungsstrukturen eine zentrale Rolle. Mit der Einführung des postbürokratischen Modells Holacracy geriet die Anschlussfähigkeit an Ansprechpartner*innen und organisationsinterne Kund*innen unter Druck. Während sich die innerorganisationale Umwelt normalerweise über die gängigen Organigramme, Bezeichnungen und Prozesse einen schnellen Überblick über andere Abteilungen verschaffen kann, gingen diese Orientierungspunkte durch die Umstellung auf das holakratische Modell verloren: »Wir waren ein weißer Fleck in der [FlatSoft Ltd.] « (Mitarbeiter Organisationsentwicklung A). In der Folge wurden vormals formale Kanäle an den Schnittstellen häufig informal weiter genutzt - auch dann, wenn im holakratischen System eigentlich neue Ansprechpartner*innen definiert wurden: »Die Auftraggeber sprechen den an, mit dem sie immer gesprochen haben « (Mitarbeiter Organisationsentwicklung B). In einem anderen Fall wurde berichtet, dass Auftraggebende verstärkt direkt auf den Lead Link zugingen, anstatt die vorgesehenen Prozesse zu nutzen. Außenstehende suchten sich in der unbekannten Struktur also formale wie informale Substitute, die sie adressieren konnten, um den Ausfall der bekannten Hierarchie und Arbeitsteilung zu kompensieren. Dadurch entstand das Bezugsproblem, die Struktur des eigenen Bereichs und die Erwartungen der relevanten innerorganisationalen Umwelt zu einem gewissen Grad in Einklang zu bringen. Besonders augenscheinlich wurde dies mit Blick auf Vertreter*innen des höheren Managements. Um das notwendige Schnittstellenmanagement zu dieser relevanten Stakeholdergruppe zu gewährleisten, schaffte die Organisationsentwicklungsabteilung eine strukturelle Lösung im Rahmen des holakratischen Modells. Mit dem sogenannten Außenminister wurde eine spezifische Rolle geschaffen, die die Schnittstellen zum Top Management beispielsweise im Konfliktfall bedienen sollte. Um dabei die nötige Anschlussfähigkeit sicherzustellen, wurde, den Erwartungen der relevanten Umwelt entsprechend, die Rolle mit dem ehemaligen und in der Formalstruktur der Gesamtorganisation weiterhin faktischen - Abteilungsleiter besetzt, wodurch die vorher bestehenden und formal von außen erwartbaren Kontakte aufrechterhalten wurden: $\gg$ Nach außen war ich immer noch der $>$ Head of $<$. Wir konnten darstellen, dass sich für die Kunden nichts ändert « (Abteilungsleiter Organisationsentwicklung). Auf diese Weise wurde den relevanten Umwelterwartungen Rechnung getragen, mit dem Folgeproblem, dass sich die bereichsinterne Komplexität, beispielsweise mit Blick auf Koordinationsbedarfe, erhöhte. 


\subsection{Die informale Reetablierung von Hierarchie}

Dass Organisationsstrukturen nicht bloß eine Frage der formalen Festlegung sind, gehört schon lange zum geteilten Wissensbestand der Organisationstheorie - das gilt auch für Hierarchie. Aus ganz unterschiedlichen Anlässen bilden sich neben der formalen Hierarchie oft auch informale Hierarchien aus. Diese zeichnen sich dadurch aus, dass sie, ohne dass über sie entschieden worden wäre, dennoch Strukturwert gewinnen und Führungschancen, ähnlich einer formalen Hierarchie, in der Organisation verteilen (Muster et al. 2020). Informale Hierarchien existieren dann als feste Erwartungsstrukturen parallel, ergänzend zur oder statt der formalen, in Organigrammen abgebildeten, Rangordnung (Diefenbach und Sillince 2011). Die Einführung von Arbeitsmodellen, die gezielt formale Hierarchie reduzieren, fördert eine solche Etablierung informaler Hierarchie - jedenfalls dann, wenn diejenigen Bezugsprobleme, die vorher durch hierarchische Strukturen gelöst wurden, nicht durch funktionale Äquivalente abgedeckt werden (vgl. Ingvaldsen und Benders 2020). Im Fallvergleich zeigen sich daher auch unterschiedliche Intensitäten informaler Strukturierung. Grundsätzlich lässt sich aber festhalten: Postbürokratische Organisationsformen scheinen die Relevanzwerdung informaler Hierarchie potenziell herauszufordern (ähnlich auch Hoering et al. 2001).

Bei der AGILETools SE zeigte sich dies auf Ebene der Assemblages. In anderen Hallen betreuten Vorarbeiter Gruppen gleicher Gewerke. In der selbstorganisierten Werkshalle dagegen wurden sogenannte Rep Links eingesetzt, die das Assemblage repräsentieren, den Informationsfluss zur Hallenleitung sicherstellen und darüber hinaus weiterhin in den Assemblages mitarbeiten sollten. Diese Funktion war allerdings nicht mit den typischen Befugnissen von Vorgesetzten verknüpft, denn weder durfte der Rep Link Urlaube oder Schichten zuteilen (das sollte konsensual im Assemblage geschehen), noch Anweisungen erteilen oder Sanktionen verhängen. Dennoch verlagerten sich mit der Zeit in manchen Assemblages die Aufgaben der Konfliktlösung, zum Beispiel zwischen konkurrierenden Wünschen der Schichtzuteilung, auf die Rep Links oder ihre Vertreter. So übernahmen sie nach und nach Aufgaben, die in anderen Hallen Vorarbeiter ausübten - allerdings ohne den formalen Auftrag dafür und ohne mit den entsprechenden Einflussmitteln formaler Hierarchie ausgestattet zu sein. Folglich blieb der Führungsanspruch der Rep Links immer auch kontestiert. Durch die fehlenden formalen Einflussmittel blieben sie vielerorts auf den guten Willen der AssemblageMitglieder angewiesen: »Sie [die Rep Links] können nicht anweisen, sie müssen nett fragen. Da sagen auch schon einmal welche $>$ Nein $\ll \ll$ (Mitarbeiter Assemblage A). Das Bezugsproblem, für das die informale Hierarchie eine Lösung darstellt, ist die Klärung von Konflikten unter Gleichrangigen (vgl. auch Fröhlich 1983). Das entstehende Folgeproblem ist in diesem Fall die Belastung einzelner Personen, nämlich der Rep Links und ihrer Vertreter. Sie mussten unter Verzicht auf formale Einflussmittel entweder 
über informalen Tauschhandel oder durch persönliche Initiative, zum Beispiel durch Überzeugungsarbeit, für ihre Entscheidungen Gefolgschaft generieren - und zwar ohne von der formalen Hierarchie geschützt zu sein. Im Gegenteil: Im Konfliktfall hätte ihre Initiative sogar als übergriffig oder anmaßend, jedenfalls als klarer Regelverstoß rezipiert werden können. Eine alternative, funktional äquivalente und teilweise auch praktizierte Lösung stellte das Weiterreichen der ungelösten Konflikte an die Hallenleitung dar (für einen ähnlichen Fall siehe Kühl 2001b). Auch hier geriet man allerdings in Konflikt mit der formalen Erwartung, dass die Assemblages sich vorrangig selbst organisieren sollten.

Auch bei der FlatSoft Ltd. wurde von Tendenzen der Rehierarchisierung berichtet. Neben dem bereits erwähnten Druck an den Schnittstellen, gegenüber Ansprechpartner*innen als hierarchische Adressen aufzutreten, sahen sich beispielsweise manche Lead Links innerhalb ihrer Teams mit Ansprüchen konfrontiert, neben der fachlichen Führungsrolle verstärkt auch wieder klassische Aufgaben disziplinarischer Führungskräfte zu übernehmen. Als Bezugsproblem zeigte sich auch hier die Lösung von Konflikt- oder Krisensituationen sowohl in Richtung von Kund*innen als auch mit Blick auf persönliche Probleme unter Kolleg*innen: »Bei bestimmten Themen wird man als Lead Link automatisch adressiert, auch, wenn die Accountability nicht beim Lead Link liegt. Bei heiklen Themen wird man schnell zur Hilfe geholt, z. B. Konflikte mit Kunden, z. B. wenn Hilfe benötigt wird « (Lead Link A). In Summe schien die informale Wiedereinführung von Hierarchie bei der FlatSoft Ltd. allerdings eine deutlich geringere Rolle zu spielen als im Falle der AGILETools SE. Ein Grund dafür mag darin liegen, dass das Organisationsmodell Holacracy ein auf Meetings basierendes Verfahren zur Entscheidungsfindung vorsieht, das sowohl für operative wie auch organisationsgestalterische Fragen genutzt werden soll. Den Mitarbeitenden stand so von Anfang an ein funktionales Äquivalent zur Verfügung, um notwendige Entscheidungen formal herbeizuführen.

In den von uns untersuchten Fällen wird deutlich, dass sowohl an den Rändern als auch innerhalb postbürokratischer Einheiten eine Problemverschiebung stattfindet. Im Fallvergleich konnte gezeigt werden, dass konkurrierende formale Erwartungen, insbesondere an den relevanten Schnittstellen nach außen, und informale Hierarchiebildung innerhalb der stärker selbstorganisierten Einheiten erwartbare Folgeprobleme des Verzichts auf formaler Hierarchie sein können.

\section{Resümee und Ausblick}

Im vorliegenden Beitrag haben wir entlang von zwei Fällen Folgen der Einführung postbürokratischer Arbeitsweisen in Großorganisationen untersucht. Dabei zeigte sich, dass mit Blick auf den Umgang mit formaler Hierarchie Spannungsfelder sowohl an Schnittstellen zur Gesamtorganisation als auch innerhalb der betrachteten Bereiche zu- 
tage traten. Diese Spannungsfelder manifestierten sich dabei in der Konkurrenz von Erwartungsstrukturen, die in den beschriebenen Fällen auf unterschiedliche Weise bearbeitet wurden und je eigene Folgeproblemen zeitigten. Während an den Schnittstellen zwischen postbürokratischen Organisationseinheiten und der weiterhin klassisch aufgestellten Restorganisation vor allem Konkurrenzverhältnisse zwischen divergierenden formalen Erwartungen in den Fokus rückten, ließen sich innerhalb der untersuchten Bereiche Tendenzen der informalen Reetablierung von Hierarchie beobachten, die in Konkurrenz zu formalen Erwartungen der Selbstorganisation traten.

Deutlich wird dabei: Gängige Bezugsprobleme des Organisierens wie die Aufrechterhaltung von Kooperation mit anderen Bereichen oder die Lösung bereichsinterner Konflikt- und Krisensituationen können infolge der Einführung postbürokratischer Arbeitsweisen an Relevanz gewinnen und so neue Aushandlungsprozesse um die Rolle formaler wie auch informaler Hierarchie auslösen. Durch eine äquivalenzfunktionalistische Perspektive rückt somit Hierarchie - unbenommen ihrer eigenen, in der gängigen Hierarchiekritik vielfach diskutierten, Folgeprobleme - als Problemlösungsstruktur in den Fokus, insbesondere dann, wenn es an entsprechenden funktionalen Äquivalenten fehlt, und zunächst unabhängig davon, welche Beobachtungen zu außerorganisationalen Umweltentwicklungen man zugrunde legt.

Eine solche Perspektive bereichert den etablierten Diskurs um die Rolle formaler Hierarchie durch ihren vorrangig deskriptiven Zugang, der in die Lage versetzt, blinde Flecken insbesondere normativer Kritik auszuleuchten. Dagegen läuft die einseitig positive Beschreibung alternativer Modelle zu bürokratischen Organisationen Gefahr, nicht mehr ausreichend sensibel für mögliche Folgeprobleme postbürokratischer Arbeitsweisen zu sein (siehe mit Blick auf teilautonome Arbeitsgruppen auch Fröhlich 1983, 542). So zeigt sich, dass die Bearbeitung konkurrierender Erwartungen dort, wo seitens der Organisation keine entsprechenden Lösungsstrukturen bereitgestellt werden, verstärkt auf einzelne Organisationsmitglieder gelastet wird. Sie sind es dann, die die damit verbundenen Risiken formaler Sanktionierung (mit-)tragen. Wenn auch die individuellen Folgen einer solchen Diagnose außerhalb des Einzugsbereichs organisationssoziologischer Forschung liegen, kann sie damit nichtsdestotrotz dazu beitragen, die weiterführende Auseinandersetzung mit diesen Folgen postbürokratischen Organisierens durch andere Disziplinen, wie beispielsweise der Organisationspsychologie, anzuregen.

Abschließend lässt sich festhalten: Hierarchie verschwindet auch nach der Einführung postbürokratischer Arbeitsweisen nicht aus Organisationen. Vielmehr wird ihre Ausdeutung und (Re-)Etablierung Teil neuer Aushandlungsprozesse. Wie wir im vorliegenden Beitrag dargestellt haben, liegt der Grund dafür nicht zuletzt im Sozialsystem Organisation selbst, das durch seine spezifischen Eigenlogiken und Bezugsprobleme die Herausbildung und Nutzung bestimmter formaler wie informaler Lösungsmecha- 
nismen nahelegt. Damit ist nicht gesagt, dass die Etablierung insbesondere formaler Hierarchie unausweichlich wäre. Gerade eine äquivalenzfunktionalistische Perspektive kann durch die Darstellung der faktischen Realisierung von Problem-/Lösungsstrukturen darauf aufmerksam machen, welche alternativen Gestaltungspotenziale hinsichtlich spezifischer Bezugsprobleme einer Organisation bestehen. Die Ergebnisse unserer Analyse sind zudem hinsichtlich der thematisierten Bezugsprobleme durch den empirischen Ausschnitt der Einführung postbürokratischer Arbeitsweisen in einzelnen Einheiten von Großorganisationen geprägt. Ob und inwieweit sie sich darüber hinaus zur Generalisierung eignen, muss an dieser Stelle offenbleiben. Dass eine äquivalenzfunktionalistische Perspektive helfen kann, eine solche Generalisierung anzuleiten, haben wir, so unsere Hoffnung, demonstrieren können.

\section{Anmerkungen}

1 Der Begriff der Postbürokratie subsummiert eine ganze Reihe von Konzepten und Modellen, die kaum ein einheitliches Begriffsverständnis abbilden. Die entsprechenden Diskursbeiträge werden vielmehr durch ihre gemeinsame Abgrenzung, vorrangig vom Weber'schen Idealtyp der Bürokratie (Weber 1972), geklammert (Oberg und Walgenbach 2007, 170ff.; Lee und Edmondson 2017, 42; siehe kritisch zu dieser Typenbildung durch Kontrastierung Barley und Kunda 2001, 77f.; Koch 2003, 302ff.). Wir verwenden den Terminus im Folgendem in diesem Sinne als Sammelbezeichnung, nicht zuletzt auch aus der Beobachtung heraus, dass der Begriff der »post-bureaucracy« den Diskurs maßgeblich prägt (McSweeney 2017, 20).

2 Im Folgenden liegt der Fokus auf der Kritik bürokratischer Organisationsstrukturen, insbesondere, wenn auch nicht ausschließlich, in Unternehmen. Davon zu unterscheiden sind unterschiedliche Formen der Bürokratiekritik, die mit Blick auf staatliche Verwaltungen formuliert werden (für einen kurzen Überblick dieser Kritikstränge siehe Derlien et al. 2011, 228ff.). Nichtsdestotrotz lässt sich auch eine Diskussion zur Übertragbarkeit der entsprechenden Konzepte und Diskurse in den Bereich staatlicher Verwaltungen beobachten (siehe für einen groben Überblick Rölle 2020; für eine allgemeine Kritik der Übertragbarkeit von Managementmoden auf Verwaltungen siehe Jansen 2020).

3 Die Kritik bezieht sich dabei zunächst häufig allgemeiner auf Bürokratie an sich. Alle skizzierten Phänomene werden dabei aber direkt mit formaler Hierarchie in Verbindung gebracht. Nicht selten scheint es, als ob Hierarchie, als ein Kernmerkmal von Bürokratien, dabei nahezu synonym zu diesen verwendet und diskutiert wird (vgl. Diefenbach und Sillince 2011, 1517).

4 Dabei wird in aller Regel übersehen, dass gerade auch aus hierarchischer Unterordnung ganz eigene Einflusschancen erwachsen. Luhmann (2018b) hat dafür den Begriff der Unterwachung geprägt. Siehe auch Kühl (2001b), der darauf verweist, dass die Bereitschaft zur Erfüllung nicht-anweisbarer Arbeitsanforderungen, also z. B. die Abweichung von formalen Regeln, gerade gegenüber der Hierarchie eine Machtquelle darstellt. Die Vergrößerung von Handlungsspielräumen, z. B. durch die Umstellung von festen Arbeitsabläufen hin zu Zielvorgaben, kann den Einfluss von Mitarbeitenden gegenüber Vorgesetzten daher im Endeffekt sogar verringern (ebd., $211 \mathrm{ff}$.).

5 Siehe kritisch dazu Maravelias (2003), der darauf hinweist, dass gerade die Trennung von Organisationsrolle und Person ein zentrales Merkmal klassisch-bürokratischer Organisationen 
sei und dass eben diese Trennung in postbürokratischen Kontexten tendenziell aufgeweicht werde. So kann gerade der Bezug auf Hierarchie und damit auf die Mitgliedschaftsrolle der Entpersonalisierung von Entscheidungen dienen (Kühl 2002, 200).

6 Der Versuch, innerorganisationalen Wandel aus veränderten, übergreifenden Umweltentwicklungen zu erklären, kann dabei durchaus kritisch betrachtet werden. Unabhängig vom Wahrheitswert der Umweltdiagnosen läuft eine solche Argumentation Gefahr, organisationsinterne Spezifika zu unterschätzen, wie Barley und Kunda $(2001,79)$ mit Blick auf Diagnosen der Verbindung neuer Organisationsformen und technologischem Wandel festhalten: »Thus, whether and how a digital technology affects the way an organization is structured depends on how the technology is designed, the way it is deployed, and how it is used and interpreted in a specific organizational context."

7 Das Erkenntnisinteresse des Forschungsprojekts lag dabei in der Beschreibung und Analyse der faktischen Realisierung postbürokratischer Arbeitsweisen in den untersuchten Organisationen und nicht in einer Diagnose zu Erfolgs- und Gelingensbedingungen der Einführung und Umsetzung entsprechender Modelle und Konzepte. Fragen zu Begleitmaßnahmen und -bedingungen wurden daher nicht systematisch erhoben und entsprechend des Forschungsinteresses nicht in die vorliegende Analyse aufgenommen.

8 Alle Namen und Angaben zu Unternehmen und Gesprächspartner*innen sind selbstverständlich anonymisiert.

9 Im Wesentlichen entsprach das Konzept dabei dem der teilautonomen Arbeitsgruppen, das bereits seit der Debatte um die »Humanisierung des Arbeitslebens« in den 1970er Jahren in wechselnder Intensität diskutiert wird und das sich insbesondere durch die Merkmale von Arbeitserweiterung, Arbeitsbereicherung und der verstärkten Möglichkeit von Arbeitswechseln auszeichnet (vgl. Antoni 1996, 25ff.).

\section{Literatur}

Adler, Paul S. 2001. »Market, Hierarchy, and Trust: The Knowledge Economy and the Future of Capitalism«. Organization Science 12 (2), 215-34. https://doi.org/10.1287/orsc.12.2.215.10117.

Altherr, Marcel. 2019. »Die Organisation der Selbstorganisation«. In Experten führen. Modelle, Ideen und Praktiken für die Organisations- und Führungsentwicklung, hrsg. v. Peter Kels und Stephanie Kaudela-Baum, 411-26. Wiesbaden: Gabler.

Alvesson, Mats und Paul Thompson. 2006. »Post-Bureaucracy?«. In The Oxford Handbook of Work and Organization, hrsg. v. Stephen Ackroyd, Rosemary Batt, Paul Thompson und Pamela S. Tolbert, 485-507. Oxford: Oxford University Press.

Ames, Lynda J. 1995. »When Sense is Not Common: Alternatives to Hierarchy at Work«. Economic and Industrial Democracy 16 (4), 553-76. https://doi.org/10.1177/0143831X95164004.

Antoni, Conny H. 1996. Teilautonome Arbeitsgruppen. Ein Königsweg zu mehr Produktivität und einer menschengerechten Arbeit? Weinheim: Psychologie Verlags Union.

Barley, Stephen R. und Gideon Kunda. 2001. »Bringing Work Back In«. Organization Science 12 (1), 76-95. https://doi.org/10.1287/orsc.12.1.76.10122.

Bernstein, Ethan, John Bunch, Niko Canner und Michael Lee. 2016. »Beyond the Holacracy Hype«. Harvard Business Review 94 (6), 38-49.

Burns, Tom und G. M. Stalker. 1971. The Management of Innovation. 3. Aufl. London: Tavistock.

Child, John und Rita G. McGrath. 2001. Organizations Unfettered: Organizational Form in an Information-Intensive Economy. The Academy of Management Journal 44 (6), 1135-48. https://doi. org/10.5465/3069393. 
Clegg, Stewart R. 2012. »The End of Bureaucracy?«. In Reinventing Hierarchy and Bureaucracy - from the Bureau to Network Organizations (Research in the Sociology of Organizations, Vol. 35), hrsg. v. Thomas Diefenbach und Rune Todnem By, 59-84. Bingley: Emerald Group Publishing Limited.

Cyert, Richard M. und James G. March.1963. A behavioral theory of the firm. Englewood Cliffs, NJ: Prentice-Hall.

Derlien, Hans-Ulrich, Doris Böhme und Markus Heindl. 2011. Bürokratietheorie. Einführung in eine Theorie der Verwaltung. Wiesbaden: VS Verlag für Sozialwissenschaften.

Diefenbach, Thomas und John A.A. Sillince. 2011. „Formal and Informal Hierarchy in Different Types of Organization«. Organization Studies 32 (11), 1515-37. https://doi.org/10.1177/ 0170840611421254.

Foss, Nicolai J. 2003. »Selective Intervention and Internal Hybrids: Interpreting and Learning from the Rise and Decline of the Oticon Spaghetti Organization«. Organization Science 14 (3), 331-49. https://doi.org/10.1287/orsc.14.3.331.15166.

Fröhlich, Dieter. 1983. »Machtprobleme in teilautonomen Arbeitsgruppen«. In Gruppensoziologie. Perspektiven und Materialien, hrsg. v. Friedrich Neidhardt, 532-51. Opladen: Westdeutscher Verlag.

Fuchs, Peter. 2009. »Hierarchien unter Druck - ein Blick auf ihre Funktion und ihren Wandel«. In Die Organisation in unruhigen Zeiten. Über die Folgen von Strukturwandel, Veränderungsdruck und Funktionsverschiebung, hrsg. v. Ralf Wetzel, Jens Aderhold und Jana Rückert-John, 53-72. Heidelberg: Carl-Auer-Systeme.

Hales, Colin. 2002. »Bureaucracy-lite and Continuities in Managerial Work«. British Journal of Management 13 (1), 51-66. https://doi.org/10.1111/1467-8551.00222.

Heckscher, Charles. 1994. »Defining the Post-Bureaucratic Type«. In The Post-Bureaucratic Organization. New Perspectives on Organizational Change, hrsg. v. Charles Heckscher und Anne Donnellon, 14-62. Thousand Oaks (u. a.): SAGE.

Heydebrand, Wolf V. 1989. »New Organizational Forms«. Work and Occupations 16 (3), 323-57. https://doi.org/10.1177/0730888489016003004.

Hoering, Sebastian, Stefan Kühl und Alexander Schulze-Fielitz. 2001. »Homogenität und Heterogenität in der Gruppenzusammensetzung. Eine mikropolitische Studie über Entscheidungsprozesse in der Gruppenarbeit«. Arbeit 10 (4), 331-51. https://doi.org/10.1515/arbeit-2001-0406.

Ingvaldsen, Jonas A. und Jos Benders. 2020. »Back through the back door? On removing supervisors to reduce hierarchy«. Baltic Journal of Management 15 (3), 473-91. https://doi.org/10. 1108/BJM-10-2019-0359.

Jansen, Till. 2020. »Prêt-à-porter oder haute couture - Managementmoden in der Verwaltung«. In Managementmoden in der Verwaltung. Sinn und Unsinn, hrsg. v. Christian Barthel, 167-83. Wiesbaden: Gabler.

Kette, Sven und Veronika Tacke. 2015. „Systemtheorie, Organisation und Kritik«. In Systemtheorie und Differenzierungstheorie als Kritik. Perspektiven im Anschluss an Niklas Luhmann, hrsg. v. Albert Scherr, 232-65. Weinheim: Beltz Juventa.

Kieser, Alfred. 1996. »Moden \& Mythen des Organisierens«. Die Betriebswirtschaft 56 (1), 21-39.

Koch, Jochen. 2003. Organisation und Differenz. Kritik des organisationstheoretischen Diskurses der Postmoderne. Wiesbaden: Westdeutscher Verlag.

Kühl, Stefan. 1999. »Krise, Renaissance oder Umbau von Hierarchien in Unternehmen? Anmerkungen zur aktuellen Managementdiskussion«. Berliner Debatte INITIAL 10 (3), 3-17.

Kühl, Stefan. 2001a. »Zentralisierung durch Dezentralisierung. Paradoxe Effekte bei Führungsgruppen«. Kölner Zeitschrift für Soziologie und Sozialpsychologie 53 (3), 467-96. https://doi.org/10. 1007/s11577-001-0075-5. 
Kühl, Stefan. 2001b. »Über das erfolgreiche Scheitern von Gruppenarbeitsprojekten. Rezentralisierung und Rehierarchisierung in Vorreiterunternehmen der Dezentralisierung «. Zeitschrift für Soziologie 30 (3), 199-222. https://doi.org/10.1515/zfsoz-2001-0303.

Kühl, Stefan. 2002. »Jenseits der Face-to-Face-Organisation. Wachstumsprozesse in kapitalmarktorientierten Unternehmen«. Zeitschrift für Soziologie 31(3), 186-210. https://doi.org/10.1515/ zfsoz-2002-0302.

Kühl, Stefan. 2011. Organisationen. Eine sehr kurze Einführung. Wiesbaden: VS Verlag für Sozialwissenschaften.

Kühl, Stefan. 2015. Wenn die Affen den Zoo regieren. Die Tücken der flachen Hierarchien. 6., aktual. Aufl. Frankfurt a.M. (u.a.): Campus.

Laloux, Frederic. 2014. Reinventing Organizations. A Guide to Creating Organizations Inspired by the Next Stage of Human Consciousness. Brüssel: Nelson Parker.

Lee, Michael Y. und Amy C. Edmondson. 2017. "Self-Managing Organizations: Exploring the Limits of Less-Hierarchical Organizing «. Research in Organizational Behavior 37, 35-58. https://doi. org/10.1016/j.riob.2017.10.002.

Luhmann, Niklas. 1973. Zweckbegriff und Systemrationalität. Über die Funktion von Zwecken in sozialen Systemen. Frankfurt a. M.: Suhrkamp.

Luhmann, Niklas. 1995. Funktionen und Folgen formaler Organisation. 4. Aufl. Berlin: Duncker \& Humblot.

Luhmann, Niklas. 2005. »Funktionale Methode und Systemtheorie«. In Soziologische Aufklärung 1. Aufsätze zur Theorie sozialer Systeme, 7. Aufl., hrsg. v. Niklas Luhmann, 39-67. Wiesbaden: VS Verlag für Sozialwissenschaften.

Luhmann, Niklas. 2018a. »Die Bedeutung der Organisationssoziologie für Betrieb und Unternehmung«. In Schriften zur Organisation 1. Die Wirklichkeit der Organisation, hrsg. v. Ernst Lukas und Veronika Tacke, 231-53. Wiesbaden: Springer VS.

Luhmann, Niklas. 2018b. »Unterwachung. Oder die Kunst, Vorgesetzte zu lenken«. In Schriften zur Organisation 1. Die Wirklichkeit der Organisation, hrsg. v. Ernst Lukas und Veronika Tacke, 415-424. Wiesbaden: Springer VS.

Maravelias, Christian. 2003. »Post-bureaucracy - control through professional freedom«. Journal of Organizational ChangeManagement 16(5),547-66. https://doi.org/10.1108/09534810310494937.

March, James G. und Herbert A. Simon. 1958. Organizations. New York: Wiley.

McSweeney, Brendan. 2017. »A Post-Bureaucratic Age? Caricatures, Claims, and Counter-Evidence«. In Evolution of the Post-Bureaucratic Organization, hrsg. v. Pierfranco Malizia, Chiara Cannavale und Fabrizio Maimone, 19-40. Hershey, PA: IGI Global.

Moser, Michaela. 2017. Hierarchielos führen. Anforderungen an eine moderne Unternehmens- und Mitarbeiterführung. Wiesbaden: Gabler.

Muster, Judith, Stefanie Büchner, Thomas Hoebel und Tabea Koepp. 2020. »Führung als erfolgreiche Einflussnahme in kritischen Momenten. Grundzüge, Implikationen und Forschungsperspektiven«. In Managementmoden in der Verwaltung. Sinn und Unsinn, hrsg. v. Christian Barthel, 285-305. Wiesbaden: Gabler.

Oberg, Achim und Peter Walgenbach. 2007. »Post-bürokratische Organisation - Utopie und Alltag. Eine Fallstudie zur IT-gestützten Kommunikation«. Zeitschrift für Management 2 (2), 168-97. https://doi.org/10.1007/s12354-007-0008-y.

Oedzes, Jacoba J., Gerben S. Van der Vegt, Floor A. Rink und Frank Walter. 2019. »On the origins of informal hierarchy: The interactive role of formal leadership and task complexity". Journal of Organizational Behavior 40 (3), 311-24. https://doi.org/10.1002/job.2330.

Ortmann, Günther. 2017. »Flottierende Signifikanten. Über Wörter wie lean, smart und agile«. Zeitschrift für Organisationsentwicklung 2/2017, 128. 
Reihlen, Markus. 1998. Die Heterarchie als postbürokratisches Organisationsmodell der Zukunft? Arbeitsbericht Nr. 96 des Seminars für Allgemeine Betriebswirtschaftslehre, Betriebswirtschaftliche Planung und Logistik der Universität zu Köln. Köln.

Rölle, Daniel. 2020. »Agile Verwaltung«. In Handbuch Digitalisierung in Staat und Verwaltung, hrsg. v. Tanja Klenk, Frank Nullmeier und Göttrik Wewer, 137-46. Wiesbaden: Springer VS.

Robertson, Brian J. 2015. Holacracy. The New Management System for a Rapidly Changing World. New York, NY: Henry Holt.

Rothschild-Whitt, Joyce. 1979. »The Collectivist Organization: An Alternative to Rational-Bureaucratic Models«. American Sociological Review 44 (4), 509-27. https://doi.org/10.2307/2094585.

Seabright, Mark A. und Jacques Delacroix. 1996. „The Minimalist Organization as a Postbureaucratic Form. The Example of Alcoholics Anonymous«. Journal of Management Inquiry 5 (2), 140-54. https://doi.org/10.1177\%2F105649269652007.

von Groddeck, Victoria und Sylvia M. Wilz, hrsg. v. 2015. Formalität und Informalität in Organisationen. Wiesbaden: Springer VS.

Weber, Max. 1972. Wirtschaft und Gesellschaft. Grundriss der verstehenden Soziologie. 5., rev. Aufl. Tübingen: Mohr.

\section{Die Autorlnnen}

Finn-Rasmus Bull, M. A. Soziologie, ist Doktorand an der Fakultät für Soziologie der Universität Bielefeld (Arbeitsbereich Organisationssoziologie). Seine Arbeitsschwerpunkte liegen in den Bereichen Postbürokratisches Organisieren und Metaorganisationen.

Kontakt: Universität Bielefeld, Fakultät für Soziologie, Postfach 100131, 33501 Bielefeld; E-Mail: finn.bull@uni-bielefeld.de

Judith Muster, Dr., ist wissenschaftliche Mitarbeiterin am Lehrstuhl für Organisations- und Verwaltungssoziologie der Universität Potsdam. Ihre Arbeitsschwerpunkte liegen u.a. in den Bereichen Führung, Postbürokratisches Organisieren und Digitalisierung.

Kontakt: Universität Potsdam, Professur für Organisations- und Verwaltungssoziologie, August-Bebel-Str. 89, 14482 Potsdam; E-Mail: judith.muster@uni-potsdam.de 